Małgorzata Filipczak, Faculty of Mathematics and Computer Sciences, Łódź University, ul. Stefana Banacha 22, 90-238 Łódź, Poland. email:

malfil@math.uni.lodz.pl

Tomasz Filipczak, Institute of Mathematics, Łódź Technical University, ul. Wólczańska 215, 93-005 Łódź, Poland. email: tfil@math.uni.lodz.pl

\title{
ON THE COMPARISON OF DENSITY TYPE TOPOLOGIES GENERATED BY FUNCTIONS
}

\begin{abstract}
In the paper there is presented a necessary and sufficient condition to compare $f$-density topologies.
\end{abstract}

\section{Preliminaries}

Throughout the paper we shall use standard notation: $\mathbb{R}$ will be the set of real numbers, $\mathbb{N}$ the set of positive integers, $\mathcal{L}$ the family of Lebesgue measurable subsets of $\mathbb{R}$ and $|E|$ the Lebesgue measure of a measurable set $E$. A point $x \in$ $\mathbb{R}$ is a right-hand density point of a measurable set $E$ if $\lim _{h \rightarrow 0^{+}} \frac{|(x, x+h) \cap E|}{h}=1$, or equivalently if $\lim _{h \rightarrow 0^{+}} \frac{|(x, x+h) \backslash E|}{h}=0$. In the same way we define a left-hand density point of $E$. We say that $x$ is a density point of $E$ if $x$ is a right-hand density point and a left-hand density point of $E$. We will denote by $\Phi_{d}(E)$ the set of all density points of $E$ (compare [8]).

From Lebesgue Density Theorem it follows that $\left|E \triangle \Phi_{d}(E)\right|=0$ for any measurable set $E$. It is also well known $\left(\left[8\right.\right.$, Th. 22.5]) that the family $\mathcal{T}_{d}:=$ $\left\{E \in \mathcal{L}: E \subset \Phi_{d}(E)\right\}$ forms a topology on the real line, called the density topology.

Mathematical Reviews subject classification: Primary: 54A10, 26A15, 26E25

Key words: density points, density topology, comparison of topologies, $f$-density

Received by the editors May 25, 2010

Communicated by: Emma D'Aniello 
Replacing the denominator $h$ in the definition of a density point by $f(h)$, where $f$ is a nondecreasing function tending to zero at zero, we obtain general notions of density point, density operator, and density topology. To keep a connection with the "ordinary" density we should also assume that $\liminf _{x \rightarrow 0+} \frac{f(x)}{x}<\infty$. Otherwise, for any $x \in \mathbb{R}$ and $E \in \mathcal{L}$

$$
\frac{|(x, x+h) \backslash E|}{f(h)} \leq \frac{|(x, x+h)|}{h} \cdot \frac{h}{f(h)} \underset{h \rightarrow 0+}{\longrightarrow} 0 .
$$

Let us denote by $\mathcal{A}$ the family of all nondecreasing functions $f:(0, \infty) \rightarrow$ $(0, \infty)$ such that $\lim _{x \rightarrow 0+} f(x)=0$ and $\liminf _{x \rightarrow 0+} \frac{f(x)}{x}<\infty$. Fix $f \in \mathcal{A}$, $E \in \mathcal{L}$ and $x \in \mathbb{R}$. We say that $x$ is a right-hand $f$-density point of $E$ if

$$
\lim _{h \rightarrow 0+} \frac{|(x, x+h) \backslash E|}{f(h)}=0 .
$$

By $\Phi_{f}^{+}(E)$ we denote the set of all right-hand $f$-density points of $E$. In the same way one may define left-hand $f$-density points of $E$ and the set $\Phi_{f}^{-}(E)$. We say that $x$ is an $f$-density point of $E$ if it is a right and left-hand $f$-density point of $E$. By $\Phi_{f}(E)$ we denote the set of all $f$-density points of $E$, i.e. $\Phi_{f}(E):=\Phi_{f}^{+}(E) \cap \Phi_{f}^{-}(E)$.

It is easily seen that

- $\Phi_{f}^{+}(E+a)=\Phi_{f}^{+}(E)+a$,

- $\Phi_{f}^{+}(E \cap F)=\Phi_{f}^{+}(E) \cap \Phi_{f}^{+}(F)$,

- if $|E \triangle F|=0$ then $\Phi_{f}^{+}(E)=\Phi_{f}^{+}(F)$,

and analogous properties hold for $\Phi_{f}^{-}$and $\Phi_{f}$. It is also clear that

- $x$ is an $f$-density point of $E$ if and only if

$$
\lim _{\substack{h \rightarrow 0, k \rightarrow 0 \\ h \geq 0, k \geq 0, h+k>0}} \frac{|(x-h, x+k) \backslash E|}{f(h+k)}=0 .
$$

In $[2]$ it was proved that

- $\Phi_{f}^{+}(E)\left(\Phi_{f}^{-}(E), \Phi_{f}(E)\right)$ are measurable,

- $\mathcal{T}_{f}:=\left\{E \in \mathcal{L}: E \subset \Phi_{f}(E)\right\}$ is a topology stronger than the natural topology on the real line. 
The topology $\mathcal{T}_{f}$ is called a topology generated by a function $f$ or $f$-density topology. Among $f$-density topologies there are: the density topology, topologies generated by sequences (compare [3]), $\psi$-density topologies (compare [1]) and others. The purpose of this paper is to produce necessary and sufficient conditions for the inclusion $\mathcal{T}_{f_{1}} \subset \mathcal{T}_{f_{2}}$ for $f_{1}, f_{2} \in \mathcal{A}$.

Different functions can generate the same operator and the same topology. The only important thing is the behavior of a function $f \in \mathcal{A}$ close to zero. Defining a function $f$ on some interval $(0, \delta)$ we consider that it is specified in any permissible way on $[\delta, \infty)$. Obviously, even functions $f_{1}, f_{2} \in \mathcal{A}$ such that $f_{1}(x) \neq f_{2}(x)$ for all $x>0$ can generate the same operator (for example, this is true if for some $r>0, \frac{1}{r}<\frac{f_{1}(x)}{f_{2}(x)}<r$ for all $x$ sufficiently close to zero). Fortunately, different operators generate different topologies. To check if $\mathcal{T}_{f_{1}} \subset \mathcal{T}_{f_{2}}$, it is enough to examine right-hand $f$-density point at zero for all measurable sets. Indeed, from $\Phi_{f}(E+a)=\Phi_{f}(E)+a, \Phi_{f}^{-}(-E)=-\Phi_{f}^{+}(E)$ and [3, Prop. 4], it follows

Proposition 1. For each $f_{1}, f_{2} \in \mathcal{A}$ the following conditions are equivalent

$$
\begin{aligned}
& \text { 1. } \underset{E \in \mathcal{L}}{\forall}\left(0 \in \Phi_{f_{1}}^{+}(E) \Rightarrow 0 \in \Phi_{f_{2}}^{+}(E)\right), \\
& \text { 2. } \underset{E \in \mathcal{L}}{\forall}\left(0 \in \Phi_{f_{1}}(E) \Rightarrow 0 \in \Phi_{f_{2}}(E)\right), \\
& \text { 3. } \underset{E \in \mathcal{L}}{\forall} \Phi_{f_{1}}(E) \subset \Phi_{f_{2}}(E), \\
& \text { 4. } \mathcal{T}_{f_{1}} \subset \mathcal{T}_{f_{2}} .
\end{aligned}
$$

\section{Useful lemmas}

In the paper we check whether, for certain functions $f_{1}, f_{2} \in \mathcal{A}$, there exists a measurable set $E$, such that $0 \in \Phi_{f_{2}}(E) \backslash \Phi_{f_{1}}(E)$. To simplify proofs we will consider interval sets and functions "constant on intervals". We say that $E$ is an interval set if $E=\cup_{n=1}^{\infty}\left[a_{n}, b_{n}\right]$ for some tending to zero sequences $\left(a_{n}\right),\left(b_{n}\right)$ with $0<b_{n+1}<a_{n}<b_{n}, n \in \mathbb{N}$. By $\mathcal{A}_{s}$ we denote the family of all functions $f \in \mathcal{A}$ of the form

$$
f(x)=y_{n} \quad \text { for } \quad x \in\left(x_{n+1}, x_{n}\right],
$$

where sequences $\left(x_{n}\right)$ and $\left(y_{n}\right)$ are decreasing and tend to zero.

Fix an arbitrary function $f \in \mathcal{A}$. Let $a:=f(1),\left(k_{n}\right)$ be an increasing sequence of all numbers $n$ for which $f^{-1}\left(\left(\frac{a}{2^{k_{n}+1}}, \frac{a}{2^{k_{n}}}\right]\right)$ is a nondegenerated interval and let $x_{n}$ be the right endpoint of this interval. In [3, Th. 1] it was 
demonstrated that the function $g(x):=\frac{a}{2^{k_{n}}}$ for $x \in\left(x_{n+1}, x_{n}\right]$ belongs to $\mathcal{A}_{s}$ and $\Phi_{f}=\Phi_{g}$. Thus we have

Lemma 1. For any function $f \in \mathcal{A}$ there is a function $g \in \mathcal{A}_{s}$ such that $\Phi_{f}=\Phi_{g}, g \leq 2 f$ and $f(x) \leq g(x)$ except for a countable set.

Lemma 2. Let $f \in \mathcal{A}_{s}, t, h$ be positive numbers and $E$ be a measurable set satisfying

$$
\limsup _{x \rightarrow 0+} \frac{|E \cap(0, x)|}{f(x)}>t .
$$

There is an interval $[a, b] \subset(0, h)$ such that

$$
\frac{|E \cap(a, b)|}{f(b)}=t \quad \text { and } \quad \frac{|E \cap(a, x)|}{f(x)} \leq t \text { for } x \in(a, b] .
$$

Proof. Let $\left(x_{n}\right)$ and $\left(y_{n}\right)$ be decreasing sequences tending to 0 such that $f(x)=y_{n}$ for $x \in\left(x_{n+1}, x_{n}\right]$. Since $\frac{|E \cap(0, x)|}{f(x)} \leq \frac{\left|E \cap\left(0, x_{n}\right)\right|}{f\left(x_{n}\right)}$ for $x \in\left(x_{n+1}, x_{n}\right]$,

$$
\limsup _{n \rightarrow \infty} \frac{\left|E \cap\left(0, x_{n}\right)\right|}{f\left(x_{n}\right)}=\limsup _{x \rightarrow 0+} \frac{|E \cap(0, x)|}{f(x)}>t .
$$

Fix a positive integer $n_{0}$ such that $x_{n_{0}}<h$ and $\frac{\left|E \cap\left(0, x_{n_{0}}\right)\right|}{f\left(x_{n_{0}}\right)}>t$. From the continuity of Lebesgue measure, it follows that $\frac{\left|E \cap\left(c, x_{n_{0}}\right)\right|}{f\left(x_{n_{0}}\right)}=t$ for some $c \in$ $\left(0, x_{n_{0}}\right)$. Let

$$
p:=\max \left\{n: n \geq n_{0} \wedge x_{n}>c \wedge \frac{\left|E \cap\left(c, x_{n}\right)\right|}{f\left(x_{n}\right)} \geq t\right\}
$$

and $b:=x_{p}$. Using again the continuity of measure we find $a \in[c, b]$ with

$$
\frac{|E \cap(a, b)|}{f(b)}=t .
$$

Thus, for $x \in(a, b] \cap\left(x_{p+1}, x_{p}\right]$,

$$
\frac{|E \cap(a, x)|}{f(x)} \leq \frac{\left|E \cap\left(a, x_{p}\right)\right|}{f\left(x_{p}\right)}=t .
$$

On the other hand, if $x \in(a, b] \cap\left(a, x_{p+1}\right]$, then $x \in\left(x_{n+1}, x_{n}\right]$ for some $n>p$. Since $x_{n}>a \geq c$, we have $\frac{\left|E \cap\left(c, x_{n}\right)\right|}{f\left(x_{n}\right)}<t$ and

$$
\frac{|E \cap(a, x)|}{f(x)} \leq \frac{\left|E \cap\left(c, x_{n}\right)\right|}{f\left(x_{n}\right)}<t .
$$


Lemma 3. For any $f \in \mathcal{A}_{s}$ there exists an interval set $C$ and a positive number $t$ such that

$$
t \leq \limsup _{x \rightarrow 0+} \frac{|C \cap(0, x)|}{f(x)} \leq 2 t .
$$

Proof. Let $\left(x_{n}\right)$ and $\left(y_{n}\right)$ be decreasing sequences tending to 0 such that $f(x)=y_{n}$ for $x \in\left(x_{n+1}, x_{n}\right]$. Since $f \in \mathcal{A}$, there is $t>0$ such that $\limsup _{x \rightarrow 0+} \frac{|\mathbb{R} \cap(0, x)|}{f(x)}=\limsup \sup _{x \rightarrow 0+} \frac{x}{f(x)}>t$. Applying Lemma 2 we can choose sequences $\left(a_{n}\right),\left(b_{n}\right)$ such that $a_{n}<b_{n}, b_{n+1}<\min \left\{\frac{1}{n} a_{n}, t f\left(a_{n}\right)\right\}$,

$$
\frac{b_{n}-a_{n}}{f\left(b_{n}\right)}=t \text { and } \frac{x-a_{n}}{f(x)} \leq t \text { for } x \in\left(a_{n}, b_{n}\right] .
$$

Put $C:=\bigcup_{n=1}^{\infty}\left[a_{n}, b_{n}\right]$. For any $n \in \mathbb{N}$ we have $\frac{\left|C \cap\left(0, b_{n}\right)\right|}{f\left(b_{n}\right)}>\frac{b_{n}-a_{n}}{f\left(b_{n}\right)}=t$, and consequently $\lim \sup _{x \rightarrow 0+} \frac{|C \cap(0, x)|}{f(x)} \geq t$. On the other hand, if $x \in\left(a_{n}, b_{n}\right]$ then

$$
\frac{|C \cap(0, x)|}{f(x)} \leq \frac{x-a_{n}+b_{n+1}}{f(x)} \leq \frac{x-a_{n}}{f(x)}+\frac{b_{n+1}}{f\left(a_{n}\right)}<t+t=2 t,
$$

and if $x \in\left(b_{n}, a_{n-1}\right]$ then applying the previous result we obtain

$$
\frac{|C \cap(0, x)|}{f(x)}=\frac{\left|C \cap\left(0, b_{n}\right)\right|}{f(x)} \leq \frac{\left|C \cap\left(0, b_{n}\right)\right|}{f\left(b_{n}\right)}<2 t .
$$

Hence $\lim \sup _{x \rightarrow 0+} \frac{|C \cap(0, x)|}{f(x)} \leq 2 t$.

\section{Comparison of topologies}

By definition, from $\lim \sup _{x \rightarrow 0^{+}} \frac{f_{1}(x)}{f_{2}(x)}<\infty$ it follows that $\Phi_{f_{1}} \subset \Phi_{f_{2}}$ and $\mathcal{T}_{f_{1}} \subset \mathcal{T}_{f_{2}}$. Consequently, if $\liminf \inf _{x \rightarrow 0^{+}} \frac{f_{1}(x)}{f_{2}(x)}>0$ and $\lim \sup _{x \rightarrow 0^{+}} \frac{f_{1}(x)}{f_{2}(x)}<\infty$, then $\mathcal{T}_{f_{1}}=\mathcal{T}_{f_{2}}$. However, the functions

$$
f_{1}(x):=\frac{1}{n !} \text { for } x \in\left(\frac{1}{(n+1) !}, \frac{1}{n !}\right], n \in \mathbb{N}
$$

and

$$
f_{2}(x):=\frac{1}{n !} \text { for } x \in\left[\frac{1}{(n+1) !}, \frac{1}{n !}\right), n \in \mathbb{N}
$$

generate the same topology (see [3, Ex. 1]), although $\liminf _{x \rightarrow 0^{+}} \frac{f_{1}(x)}{f_{2}(x)}=0$. 
Theorem 1. (compare [10, Th. 2.6]) For any functions $f_{1}, f_{2} \in \mathcal{A}$ with $\lim _{x \rightarrow 0+} \frac{f_{1}(x)}{f_{2}(x)}=0$ there is a measurable set $D$ such that $0 \in \Phi_{f_{2}}(D) \backslash \Phi_{f_{1}}(D)$.

Proof. By Lemma 1, there is a function $g_{1} \in \mathcal{A}_{s}$ such that $g_{1} \leq 2 f_{1}$ and $\Phi_{f_{1}}=\Phi_{g_{1}}$. Using Lemma 3 we can find an interval set $C$ and a positive number $t$ such that

$$
t \leq \limsup _{x \rightarrow 0+} \frac{|C \cap(0, x)|}{g_{1}(x)} \leq 2 t .
$$

Let $D:=\mathbb{R} \backslash C$. Since

$$
\limsup _{x \rightarrow 0+} \frac{|(0, x) \backslash D|}{g_{1}(x)}=\limsup _{x \rightarrow 0+} \frac{|C \cap(0, x)|}{g_{1}(x)} \geq t>0,
$$

we have $0 \notin \Phi_{g_{1}}(D)=\Phi_{f_{1}}(D)$. On the other hand,

$$
\begin{aligned}
\limsup _{x \rightarrow 0+} \frac{|(0, x) \backslash D|}{f_{2}(x)} & \leq \limsup _{x \rightarrow 0+} \frac{|(0, x) \backslash D|}{f_{1}(x)} \cdot \limsup _{x \rightarrow 0+} \frac{f_{1}(x)}{f_{2}(x)} \\
& \leq 2 \limsup _{x \rightarrow 0+} \frac{|C \cap(0, x)|}{g_{1}(x)} \cdot \limsup _{x \rightarrow 0+} \frac{f_{1}(x)}{f_{2}(x)} \leq 4 t \cdot 0=0,
\end{aligned}
$$

so $0 \in \Phi_{f_{2}}(D)$.

Corollary 1. If $\lim _{x \rightarrow 0+} \frac{f_{1}(x)}{f_{2}(x)}=0$, then $\mathcal{T}_{f_{1}} \varsubsetneqq \mathcal{T}_{f_{2}}$.

It is easily seen that the condition $\lim _{x \rightarrow 0+} \frac{f_{1}(x)}{f_{2}(x)}=0$ is not necessary for $\mathcal{T}_{f_{1}} \varsubsetneqq \mathcal{T}_{f_{2}}$. Indeed, if $f_{1}(x):=x$ and $f_{2}(x):=\frac{1}{n !}$ for $x \in\left(\frac{1}{(n+1) !}, \frac{1}{n !}\right]$, then $\lim \sup _{x \rightarrow 0+} \frac{f_{1}(x)}{f_{2}(x)}=1>0$ and $\mathcal{T}_{f_{1}}=\mathcal{T}_{d} \varsubsetneqq \mathcal{T}_{f_{2}}$ (see [3, Th. 4]).

S. J. Taylor in [9] investigated $f$-density points for a function $f(x)=$ $x \psi(x)$, where $\psi$ is a nondecreasing and continuous function with $\lim _{x \rightarrow 0+} \psi(x)=$ 0 . The symmetric version of Taylor's definition was used in [10] to introduce a notion of $\psi$-density point and $\psi$-density topology. A main result of [11] gave necessary and sufficient conditions for the the inclusion of one $\psi$-density topology in another. We will transfer it to $f$-density topologies. Fortunately, our general framework allows for a simplification of proofs given in [11].

Let $f_{1}, f_{2} \in \mathcal{A}$. We define sequences

$$
\begin{aligned}
& A_{n}:=A_{n f_{1} f_{2}}:=\left\{x \in(0, \infty): f_{1}(x)<\frac{1}{n} f_{2}(x)\right\}, \\
& \varepsilon_{n}:=\varepsilon_{n f_{1} f_{2}}:=\limsup _{x \rightarrow 0+} \frac{\left|A_{n} \cap(0, x)\right|}{f_{1}(x)} .
\end{aligned}
$$


Of course, these sequences are decreasing, and so $\left(\varepsilon_{n}\right)$ is convergent.

Lemma 4. If $0 \in \Phi_{f_{2}}^{+}(\mathbb{R} \backslash E)$, then for every positive integer $n$

$$
\limsup _{x \rightarrow 0+} \frac{|E \cap(0, x)|}{f_{1}(x)}=\limsup _{x \rightarrow 0+} \frac{\left|E \cap A_{n} \cap(0, x)\right|}{f_{1}(x)} .
$$

Proof. By assumption,

$$
\lim _{x \rightarrow 0+} \frac{|E \cap(0, x)|}{f_{2}(x)}=0 .
$$

Let us fix a positive integer $n$ and a positive $x$ such that $f_{1}(x)<1$. If $x \notin A_{n}$, then $f_{1}(x) \geq \frac{1}{n} f_{2}(x)$ and consequently

$$
\frac{\left|\left(E \backslash A_{n}\right) \cap(0, x)\right|}{f_{1}(x)} \leq \frac{n|E \cap(0, x)|}{f_{2}(x)} .
$$

If $(0, x] \subset A_{n}$, then

$$
\frac{\left|\left(E \backslash A_{n}\right) \cap(0, x)\right|}{f_{1}(x)}=0 .
$$

Finally, if $x \in A_{n}$ and $(0, x] \backslash A_{n} \neq \emptyset$, then for $b:=\sup \left((0, x] \backslash A_{n}\right)$ and any $a$ from $\left[b-b f_{1}(b), b\right] \backslash A_{n}$ we have $f_{1}(a) \geq \frac{1}{n} f_{2}(a)$, and so

$$
\begin{aligned}
\frac{\left|\left(E \backslash A_{n}\right) \cap(0, x)\right|}{f_{1}(x)} & =\frac{\left|\left(E \backslash A_{n}\right) \cap(0, b)\right|}{f_{1}(x)} \leq \frac{\left|\left(E \backslash A_{n}\right) \cap(0, a)\right|}{f_{1}(a)}+\frac{b-a}{f_{1}(b)} \\
& \leq \frac{n|E \cap(0, a)|}{f_{2}(a)}+b .
\end{aligned}
$$

Since $a \leq b \leq x$, conditions (1)-(4) imply

$$
\lim _{x \rightarrow 0+} \frac{\left|\left(E \backslash A_{n}\right) \cap(0, x)\right|}{f_{1}(x)}=0
$$

which gives

$$
\begin{aligned}
\limsup _{x \rightarrow 0+} \frac{|E \cap(0, x)|}{f_{1}(x)} & \leq \limsup _{x \rightarrow 0+} \frac{\left|E \cap A_{n} \cap(0, x)\right|}{f_{1}(x)}+\limsup _{x \rightarrow 0+} \frac{\left|\left(E \backslash A_{n}\right) \cap(0, x)\right|}{f_{1}(x)} \\
& =\limsup _{x \rightarrow 0+} \frac{\left|E \cap A_{n} \cap(0, x)\right|}{f_{1}(x)} \leq \limsup _{x \rightarrow 0+} \frac{|E \cap(0, x)|}{f_{1}(x)} .
\end{aligned}
$$


Corollary 2. If $0 \in \Phi_{f_{2}}^{+}(\mathbb{R} \backslash E)$, then for every positive integer $n$

$$
\limsup _{x \rightarrow 0+} \frac{|E \cap(0, x)|}{f_{1}(x)} \leq \limsup _{x \rightarrow 0+} \frac{\left|A_{n} \cap(0, x)\right|}{f_{1}(x)} .
$$

Theorem 2. Let $f_{1}, f_{2} \in \mathcal{A}$. If $\lim _{n \rightarrow \infty} \varepsilon_{n}=0$, then $\mathcal{T}_{f_{2}} \subset \mathcal{T}_{f_{1}}$.

Proof. Let us suppose that $0 \in \Phi_{f_{2}}^{+}(E)$. By Corollary 2,

$$
0 \leq \limsup _{x \rightarrow 0+} \frac{|(0, x) \backslash E|}{f_{1}(x)} \leq \limsup _{x \rightarrow 0+} \frac{\left|A_{n} \cap(0, x)\right|}{f_{1}(x)}=\varepsilon_{n}
$$

for every positive integer $n$. Thus $\lim \sup _{x \rightarrow 0+} \frac{|(0, x) \backslash E|}{f_{1}(x)}=0$, and consequently $0 \in \Phi_{f_{1}}^{+}(E)$. From Proposition 1 we conclude that $\mathcal{T}_{f_{2}} \subset \mathcal{T}_{f_{1}}$.

Theorem 3. Let $f_{1}, f_{2} \in \mathcal{A}$. If $\lim _{n \rightarrow \infty} \varepsilon_{n}>0$, then there is a measurable set $D$ such that $0 \in \Phi_{f_{2}}(D) \backslash \Phi_{f_{1}}(D)$.

Proof. By Lemma 1 there exists $g_{1} \in \mathcal{A}_{s}$ such that $\Phi_{f_{1}}=\Phi_{g_{1}}$ and $g_{1} \leq 2 f_{1}$. Let us define

$$
\widetilde{A}_{n}:=\left\{x \in(0, \infty): g_{1}(x)<\frac{1}{n} f_{2}(x)\right\} \text { and } \widetilde{\varepsilon}_{n}:=\limsup _{x \rightarrow 0+} \frac{\left|\widetilde{A}_{n} \cap(0, x)\right|}{g_{1}(x)} .
$$

Since $A_{2 n} \subset \widetilde{A}_{n}$,

$$
\widetilde{\varepsilon}_{n}=\limsup _{x \rightarrow 0+} \frac{\left|\widetilde{A}_{n} \cap(0, x)\right|}{g_{1}(x)} \geq \limsup _{x \rightarrow 0+} \frac{\left|A_{2 n} \cap(0, x)\right|}{2 f_{1}(x)}=\frac{1}{2} \varepsilon_{2 n},
$$

and consequently $\lim _{n \rightarrow \infty} \widetilde{\varepsilon}_{n} \geq \frac{1}{2} \lim _{n \rightarrow \infty} \varepsilon_{2 n}>0$. Set $t:=\frac{1}{2} \lim _{n \rightarrow \infty} \widetilde{\varepsilon}_{n}$ and choose $n_{0}$ such that $\widetilde{\varepsilon}_{n}>t$ for $n \geq n_{0}$. Applying Lemma 2 we can define intervals $\left[a_{n}, b_{n}\right], n \geq n_{0}$ such that $b_{n+1}<\min \left\{a_{n}, \frac{1}{n} f_{2}\left(a_{n}\right)\right\}$,

$$
\frac{\left|\widetilde{A}_{n} \cap\left(a_{n}, b_{n}\right)\right|}{g_{1}\left(b_{n}\right)}=t \text { and } \frac{\left|\widetilde{A}_{n} \cap\left(a_{n}, x\right)\right|}{g_{1}(x)} \leq t \text { for } x \in\left(a_{n}, b_{n}\right] \text {. }
$$

Let

$$
E:=\bigcup_{n=n_{0}}^{\infty}\left(\widetilde{A}_{n} \cap\left(a_{n}, b_{n}\right)\right) \text { and } D:=\mathbb{R} \backslash E .
$$

For $n \geq n_{0}$ we have $\frac{\left|E \cap\left(0, b_{n}\right)\right|}{g_{1}\left(b_{n}\right)} \geq \frac{\left|\widetilde{A}_{n} \cap\left(a_{n}, b_{n}\right)\right|}{g_{1}\left(b_{n}\right)}=t>0$, and so $0 \notin \Phi_{g_{1}}(D)=$ $\Phi_{f_{1}}(D)$. 
It remains to prove that $0 \in \Phi_{f_{2}}(D)$. Consider $x \in\left(0, b_{n_{0}}\right]$. We first assume that $x \in\left(a_{n}, b_{n}\right]$ for some $n \geq n_{0}$ and define

$$
x^{\prime}:=\inf \left\{y:\left|\widetilde{A}_{n} \cap[y, x]\right|=0\right\} .
$$

If $x^{\prime}>a_{n}$, then one can find $x^{*} \in \widetilde{A}_{n} \cap\left(a_{n}, x^{\prime}\right]$ such that $x^{\prime}-x^{*}<g_{1}\left(a_{n}\right)$. Thus

$$
\begin{aligned}
\frac{|E \cap(0, x)|}{f_{2}(x)} & \leq \frac{\left|\widetilde{A}_{n} \cap\left(a_{n}, x\right)\right|+b_{n+1}}{f_{2}(x)} \leq \frac{\left|\widetilde{A}_{n} \cap\left(a_{n}, x^{\prime}\right)\right|}{f_{2}\left(x^{*}\right)}+\frac{b_{n+1}}{f_{2}\left(a_{n}\right)} \\
& <\frac{\left|\widetilde{A}_{n} \cap\left(a_{n}, x^{*}\right)\right|+\left(x^{\prime}-x^{*}\right)}{n g_{1}\left(x^{*}\right)}+\frac{1}{n} \leq \frac{t}{n}+\frac{1}{n}+\frac{1}{n}=\frac{t+2}{n} .
\end{aligned}
$$

If $x^{\prime} \leq a_{n}$, since $\left|\widetilde{A}_{n+1} \cap\left(a_{n+1}, b_{n+1}\right)\right|>0$, we can apply (5) for $n+1$ and obtain

$$
\frac{|E \cap(0, x)|}{f_{2}(x)}=\frac{\left|E \cap\left(0, b_{n+1}\right)\right|}{f_{2}(x)} \leq \frac{\left|E \cap\left(0, b_{n+1}\right)\right|}{f_{2}\left(b_{n+1}\right)}<\frac{t+2}{n+1} .
$$

Assume now that $x \in\left(b_{n+1}, a_{n}\right]$ for some $n \geq n_{0}$. As in the previous case, we get

$$
\frac{|E \cap(0, x)|}{f_{2}(x)} \leq \frac{\left|E \cap\left(0, b_{n+1}\right)\right|}{f_{2}\left(b_{n+1}\right)}<\frac{t+2}{n+1}
$$

From (5)-(7) it follows that $\limsup _{x \rightarrow 0+} \frac{|E \cap(0, x)|}{f_{2}(x)}=0$, which gives $0 \in \Phi_{f_{2}}(D)$.

Corollary 3. If $\mathcal{T}_{f_{2}} \subset \mathcal{T}_{f_{1}}$, then $\lim _{n \rightarrow \infty} \varepsilon_{n}=0$.

Theorem 2 and Corollary 3 lead to

Theorem 4. $\mathcal{T}_{f_{2}} \subset \mathcal{T}_{f_{1}}$ if and only if $\lim _{n \rightarrow \infty} \varepsilon_{n}=0$.

We can use the above theorem to compare the density topology $\mathcal{T}_{d}$ with others $f$-density topologies. A topology $\mathcal{T}_{f}$ is stronger than $\mathcal{T}_{d}$ if and only if for any positive $\varepsilon$ and sufficiently large $n$, the inequality

$$
\frac{\left|\left\{x \in(0, h): f(x)<\frac{x}{n}\right\}\right|}{f(h)}<\varepsilon
$$


holds for sufficiently small $h$. Analogously, $\mathcal{T}_{f} \subset \mathcal{T}_{d}$ if and only if for any $\varepsilon>0$ and sufficiently large $n$,

$$
\frac{|\{x \in(0, h): f(x)>n x\}|}{h}<\varepsilon
$$

for sufficiently small $h$.

\section{Applications}

Using the condition formulated in Theorem 4 we have found answers for some questions and have simplified some difficult proofs. Let $\langle s\rangle=\left\{s_{n}\right\}_{n \in \mathbb{N}}$ be an unbounded, nondecreasing sequence of positive numbers. We say that $x \in \mathbb{R}$ is an $\langle s\rangle$-density point of a set $E \in \mathcal{L}$ if

$$
\lim _{n \rightarrow \infty} \frac{\left|E \cap\left[x-\frac{1}{s_{n}}, x+\frac{1}{s_{n}}\right]\right|}{\frac{2}{s_{n}}}=1 .
$$

For any $E \in \mathcal{L}$ we denote by $\Phi_{\langle s\rangle}(E)$ the set of all $\langle s\rangle$-density points $E$. The family $\mathcal{T}_{\langle s\rangle}:=\left\{E \in \mathcal{L}: E \subset \Phi_{\langle s\rangle}(E\}\right.$ is a topology called topology generated by the sequence $\langle s\rangle$ (see [6]). It is easy to check that $\mathcal{T}_{\langle s\rangle}$ is equal to the topology generated by the function $f_{\langle s\rangle}(x):=\frac{1}{s_{n}}$ for $x \in\left(\frac{1}{s_{n+1}}, \frac{1}{s_{n}}\right]$, and that $f_{\langle s\rangle}$ belongs to the family $\mathcal{A}^{1}:=\left\{f \in \mathcal{A}: \liminf _{x \rightarrow 0+} \frac{f(x)}{x}>0\right\}$ (compare [3]). In [4] it is proved that there are continuum topologies $\mathcal{T}_{f}$ with $f \in \mathcal{A}_{1}$ such that $\mathcal{T}_{f}$ is different from all topologies generated by sequences. However, the construction presented in this paper is rather complicated. Theorem 4 lets us find simpler examples (compare [5]). In [6] a necessary and sufficient condition was given for comparability of density topologies generated by sequences. This condition can be easily obtained from Theorem 4. Finally, in [7] the condition from Theorem 4 is used to compare $\psi$-density topologies with topologies generated by functions from $\mathcal{A}^{0}:=\left\{f \in \mathcal{A}: \liminf \operatorname{in}_{x \rightarrow 0+} \frac{f(x)}{x}=0\right\}$ more precisely than in [1].

\section{References}

[1] M. Filipczak, T. Filipczak, Remarks on $f$-density and $\psi$-density, Tatra Mt. Math. Publ., 34 (2006), 141-149.

[2] M. Filipczak, T. Filipczak, On f-density topologies, Topology Appl., 155(17-18) (2008), 1980-1989. 
[3] M. Filipczak, T. Filipczak, Density topologies generated by functions and by sequences, Tatra Mt. Math. Publ., 40 (2008), 103-115.

[4] M. Filipczak, T. Filipczak, On the comparison of the density type topologies generated by sequences and by functions, Comm. Math., 49(2) (2009), 161-169.

[5] M. Filipczak, T. Filipczak, $\left(\Delta_{2}\right)$ condition for $f$-density topologies, submitted.

[6] M. Filipczak, T. Filipczak, J. Hejduk, On the Comparison of the Density Type Topologies, Atti Sem. Mat. Fis. Univ. Modena, 52 (2004), 37-46.

[7] T. Filipczak, The comparison of $f$-density and $\psi$-density, submitted.

[8] J. C. Oxtoby, Measure and Category, Springer-Verlag, 1980.

[9] S. J. Taylor, On strengthening the Lebesgue Density Theorem, Fund. Math., 46 (1959), 305-315.

[10] M. Terepeta, E. Wagner-Bojakowska, $\psi$-density topology, Rend. Circ. Mat. Palermo, 48 Serie II (1999), 451-476.

[11] E. Wagner-Bojakowska, W. Wilczyński, Comparison of $\psi$-density topologies, Real Analysis Exchange, 25(2) (1999-2000), 661-672. 
